\title{
Pigmented villonodular synovitis of the temporomandibular joint - computed tomography and magnetic resonance findings: a case report
}

\author{
II-Kyu Kim, Hyun-Young Cho, Hyun-Woo Cho, Ji-Hoon Seo, Dong-Hwan Lee, Wang Peng \\ Department of Oral and Maxillofacial Surgery, Inha University College of Medicine, Incheon, Korea
}

\begin{abstract}
J Korean Assoc Oral Maxillofac Surg 2014;40:140-146)
Pigmented villonodular synovitis (PVNS) is a benign but locally aggressive and destructive disease originating in the synovial membranes. It is a proliferative disorder of unknown etiology. Involvement of the temporomandibular joint (TMJ) is very rare. Computed tomography clearly reveals areas of lytic bone erosion and sclerosis, and also clearly defines the extent of the tumor which is the focal areas of hyperdensity within the soft-tissue mass. Magnetic resonance images invariably show profound hypointensity on both T1- and T2-weighted sequences due to hemosiderin pigmentation. Additionally, high signal intensity on T2-weighted images may indicate cystic loculation of the joint fluid. This case study describes a rare case of PVNS of the TMJ with bone destruction of the mandibular condyle. Complete surgical excision of the lesion was performed through a preauricular approach with temporal extension. During the 10-year follow-up, two more operations were performed due to local recurrence and the fracture of the reconstruction plate. Total joint reconstruction with Biomet was finally performed, and the absence of disease was confirmed with a biopsy report showing fibrosis with hyalinization and mild inflammation of the excised soft tissue from the old lesion.
\end{abstract}

Key words: Temporomandibular joint, Pigmented villonodular synovitis, Mandibular reconstruction

[paper submitted 2014. 4. 13 / revised 2014. 5. 27 / accepted 2014. 5. 28]

\section{Introduction}

Pigmented villonodular synovitis (PVNS) is a benign, but locally aggressive, proliferative disorder affecting the synovium, often with infiltration and/or osteoinvasion ${ }^{1-4}$. It commonly arises in the tendon sheaths and long bone joints and rarely arises in the bursae. Jaffe et al. ${ }^{1}$ introduced the term PVNS in 1941 to describe a yellow tumor-like synovial lesion. They described PVNS as both localized (affects only a portion of the joint lining or tendon sheath with a solitary pedunculated nodular lesion with no villi) and more commonly diffuse (affects the entire synovial joint membrane or bursa). The most commonly affected joint is the knee, and

\section{Il-Kyи Kim}

Department of Oral and Maxillofacial Surgery, Inha University College of Medicine, 27 Inhang-ro, Jung-gu, Incheon 400-711, Korea

TEL: +82-32-890-2470 FAX: +82-32-890-2475

E-mail: kik@inha.ac.kr

(c) This is an open-access article distributed under the terms of the Creative Commons Attribution Non-Commercial License (http://creativecommons.org/licenses/by-nc/3.0/), which permits unrestricted non-commercial use, distribution, and reproduction in any medium, provided the original work is properly cited.

Copyright (C) 2014 The Korean Association of Oral and Maxillofacial Surgeons. All rights reserved. less frequently the disease involves the hip, shoulder, ankle, or wrist ${ }^{1,2}$. Although any joint can be affected, involvement of the temporomandibular joint (TMJ) is reportedly very rare r. $^{3,4}$. Lapayowker et al. ${ }^{5}$ first described PVNS of the TMJ in 1973. According to one literature search, since 1973, a total of 45 PVNS patients with TMJ involvement have been reported to date.

In this report, we describe a rare case with 10 years of follow-up of PVNS of the TMJ that presented as an aggressive tumor with local recurrence. We also discuss and review the previous literature on PVNS of the TMJ.

\section{Case Report}

In May 2004, a 38-year-old Korean man was referred to the Department of Oral and Maxillofacial Surgery at Inha University Hospital (Incheon, Korea) with a 2-year history of painless swelling in the right preauricular area. Review of his medical history revealed schizophrenia and diabetes mellitus, which were well controlled with medication. Physical examination revealed an ill-defined, hard mass approximately $3 \times$ $4 \mathrm{~cm}$ in diameter that was mildly tender on palpation in the

This work was supported by the Inha University Research Grant. 
right preauricular area. Maximum mouth opening was limited to $30 \mathrm{~mm}$, and mandibular opening was accompanied by slight deviation to the right. There was no evidence of facial nerve-related weakness, and a history of facial trauma was not noted.

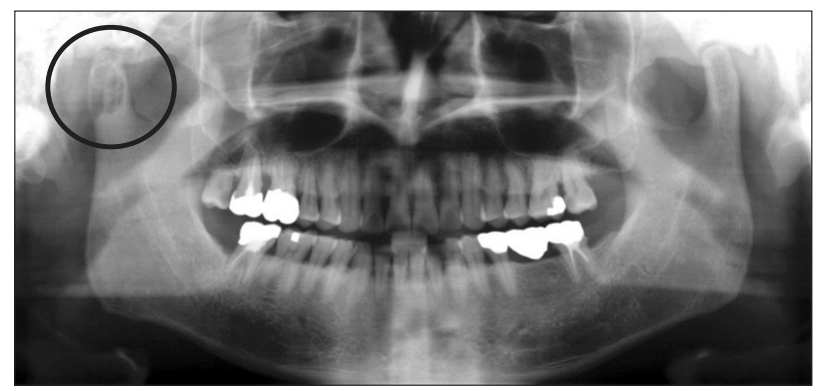

Fig. 1. The panoramic view shows the lobulated osteolytic lesion in the right condylar head.

Il-Kyu Kim et al: Pigmented villonodular synovitis of the temporomandibular joint computed tomography and magnetic resonance findings: a case report. J Korean Assoc Oral Maxillofac Surg 2014

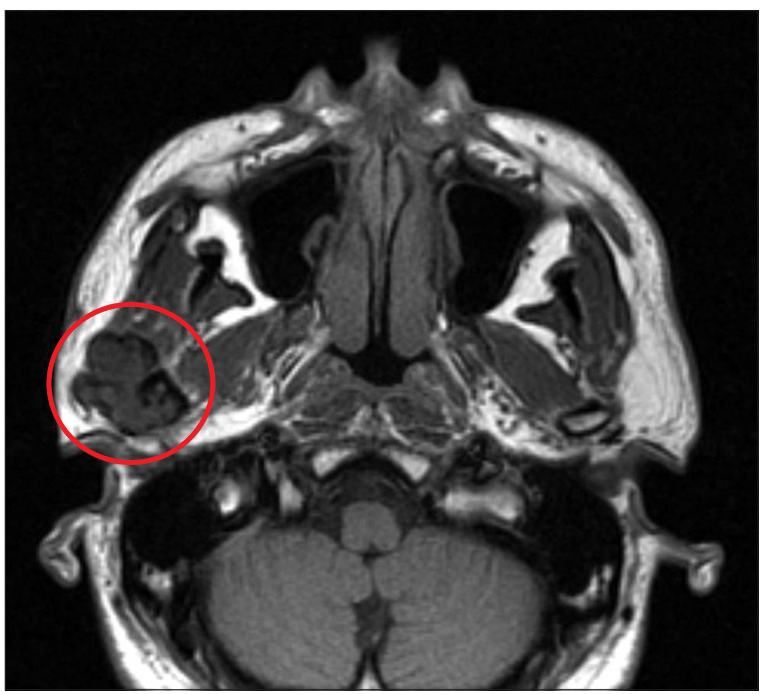

Fig. 4. The axial T1-weighted image of magnetic resonance imaging shows the intermediate signal intensity with peripheral low signal intensity rim.

Il-Kyu Kim et al: Pigmented villonodular synovitis of the temporomandibular joint computed tomography and magnetic resonance findings: a case report. J Korean Assoc Oral Maxillofac Surg 2014
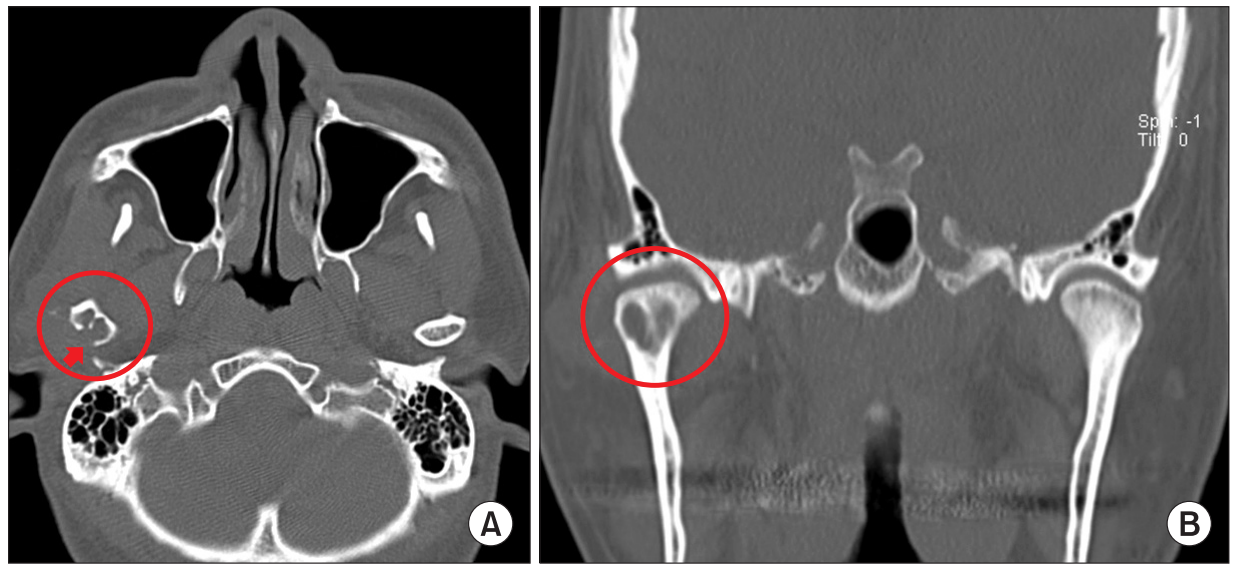

Fig. 2. The axial (A) and coronal (B) views of computed tomography show a lobulated osteolytic lesion with the bony erosion (arrow) in the mandibular condyle.

Il-Kyu Kim et al: Pigmented villonodular synovitis of the temporomandibular joint - computed tomography and magnetic resonance findings: a case report. J Korean Assoc Oral Maxillofac Surg 2014
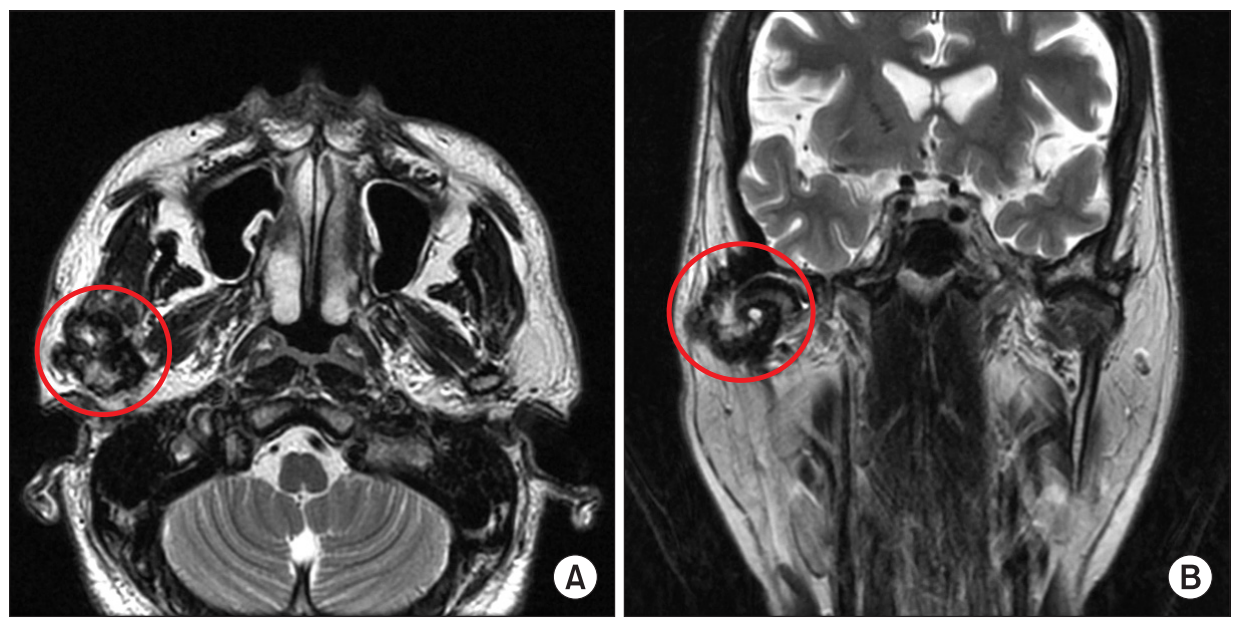

Fig. 3. The axial $(A)$ and coronal $(B)$ T2-weighted images of magnetic resonance imaging show the multilobulated mass-like lesion with inner high signal intensity and outer low signal intensity. Il-Kyu Kim et al: Pigmented villonodular synovitis of the temporomandibular joint - computed tomography and magnetic resonance findings: a case report. J Korean Assoc Oral Maxillofac Surg 2014 
A panoramic radiograph showed a lobulated cystic or osteolytic radiolucency of the right mandibular condyle.(Fig. 1) A computed tomogram (CT) showed a lobulated osteolytic and bony erosion in the right mandibular condyle.(Fig. 2) Magnetic resonance imaging (MRI) showed a lobulated-appearing enhancing soft tissue mass of the right TMJ and mandibular condyle. The $3.0 \times 2.5 \mathrm{~cm}$ irregularly shaped mass had a heterogeneous $\mathrm{T} 2$ signal intensity and isosignal intensity on T1-weighted MRI.(Figs. 3-5) Peripheral low signal intensity areas were noted on both T1- and T2- weighted magnetic resonance (MR) images. Fine needle aspiration cytology was done. The smear revealed a few clustered and scattered plump spindle cells containing hemosiderin-pigments and several scattered multinucleated giant cells.(Fig. 6) With a suspicion of PVNS, or fibrous or inflammatory pseudo-tumor of the TMJ, surgical exploration of the right TMJ, excision of the mass, and reconstruction of the mandibular condyle were also planned.

The surgery was performed under general endotracheal anesthesia. The zygomatic arch and the joint capsule were exposed by a preauricular incision with a temporal extension. A complete capsulectomy was performed for excision of the mass involving the condylar head and articular disk. The surfaces of the glenoid fossa and articular eminence were found to be normal. After reconstruction of the condyle with a titanium condylar head and plate, the surgical wound was sutured.

The excised lesion was $4.0 \times 3.0 \times 2.5 \mathrm{~cm}$ in size and in-

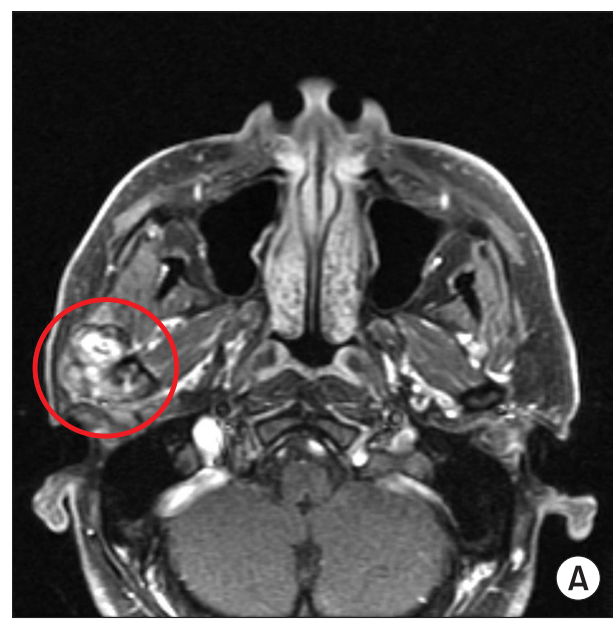

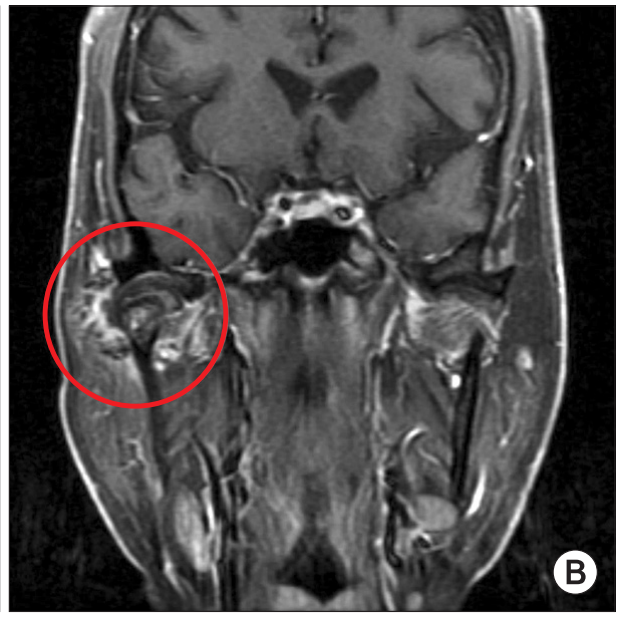

Fig. 5. The axial (A) and coronal (B) fat suppression $\mathrm{T} 1$-weighted images of magnetic resonance imaging show the multilobulated high signal intensity with the high soft tissue enhancement. Il-Kyu Kim et al: Pigmented villonodular synovitis of the temporomandibular joint - computed tomography and magnetic resonance findings: a case report. J Korean Assoc Oral Maxillofac Surg 2014

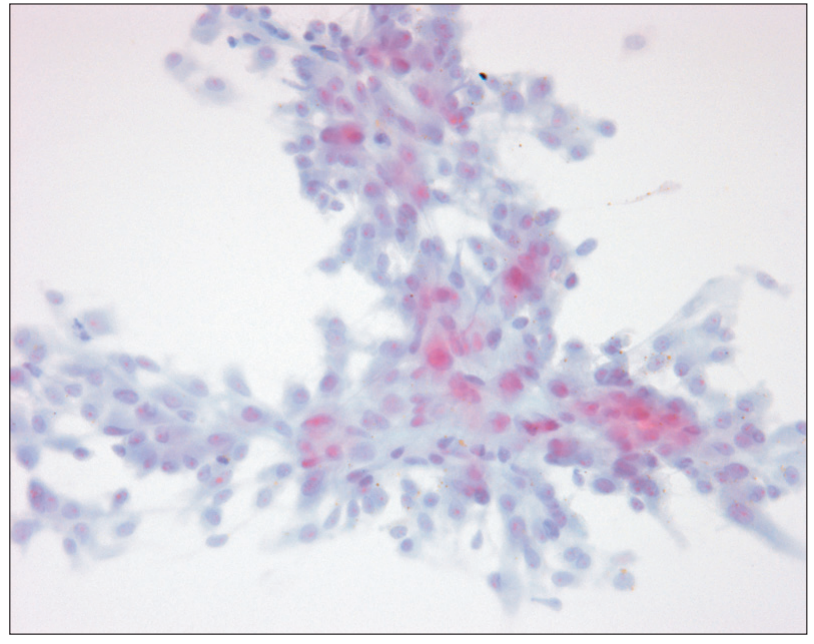

Fig. 6. Smear shows a few clustered and scattered plump spindle cells containing hemosiderin pigments (Papanicolaou staining, $\times 400)$.

Il-Kyu Kim et al: Pigmented villonodular synovitis of the temporomandibular jointcomputed tomography and magnetic resonance findings: a case report. J Korean Assoc Oral Maxillofac Surg 2014

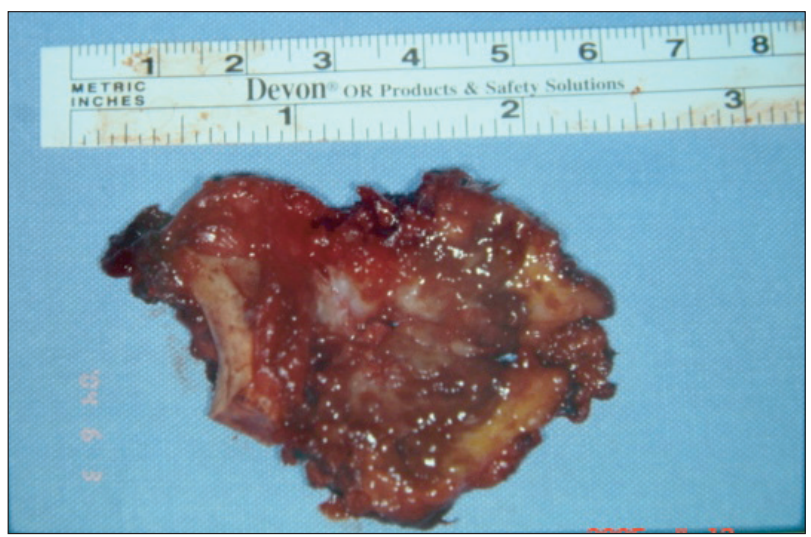

Fig. 7. Grossly, the specimen is consisted of a few fragments of brownish gray soft tissue, cartilage and bone.

Il-Kyu Kim et al: Pigmented villonodular synovitis of the temporomandibular jointcomputed tomography and magnetic resonance findings: a case report. J Korean Assoc Oral Maxillofac Surg 2014 

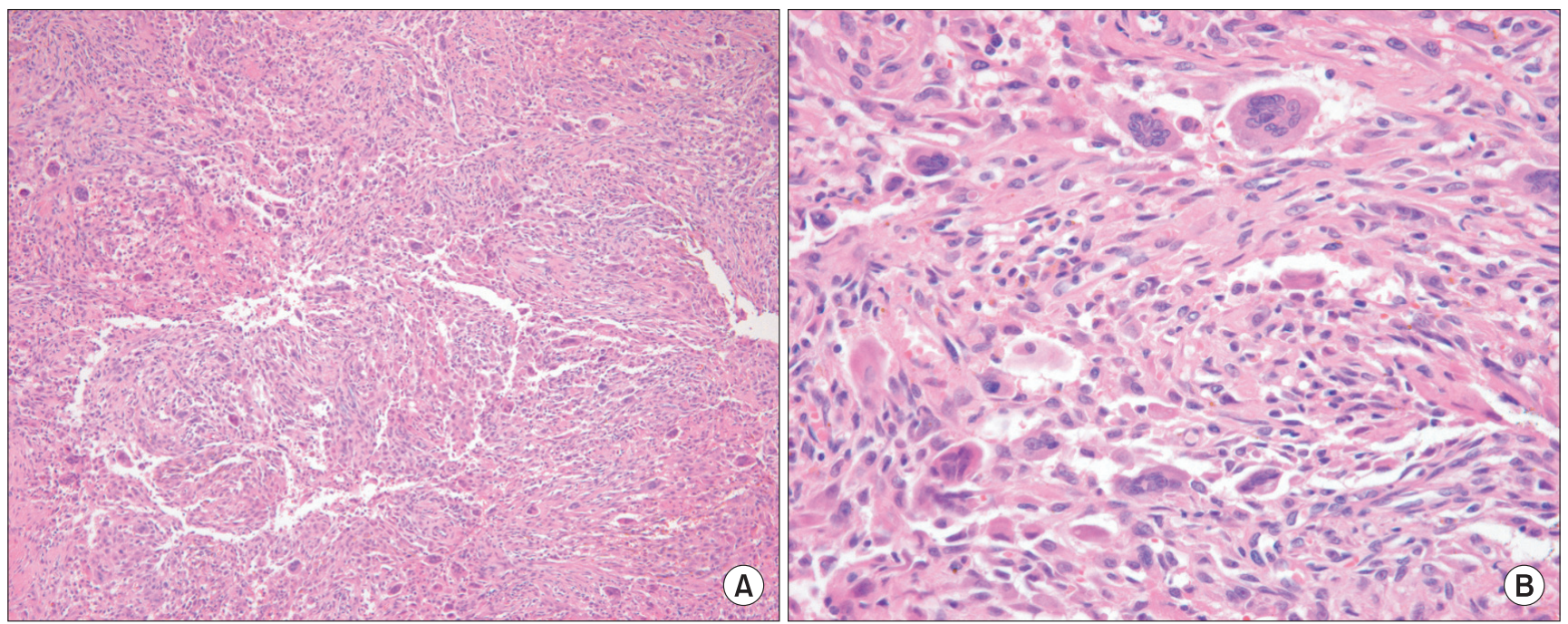

Fig. 8. The mass is composed of many mononuclear histiocytic cells and irregularly interspersed multinucleated giant cells. Hemosiderin deposits are found (H\&E staining, A: $\times 100, B: \times 400)$.

Il-Kyu Kim et al: Pigmented villonodular synovitis of the temporomandibular joint - computed tomography and magnetic resonance findings: a case report. J Korean Assoc Oral Maxillofac Surg 2014
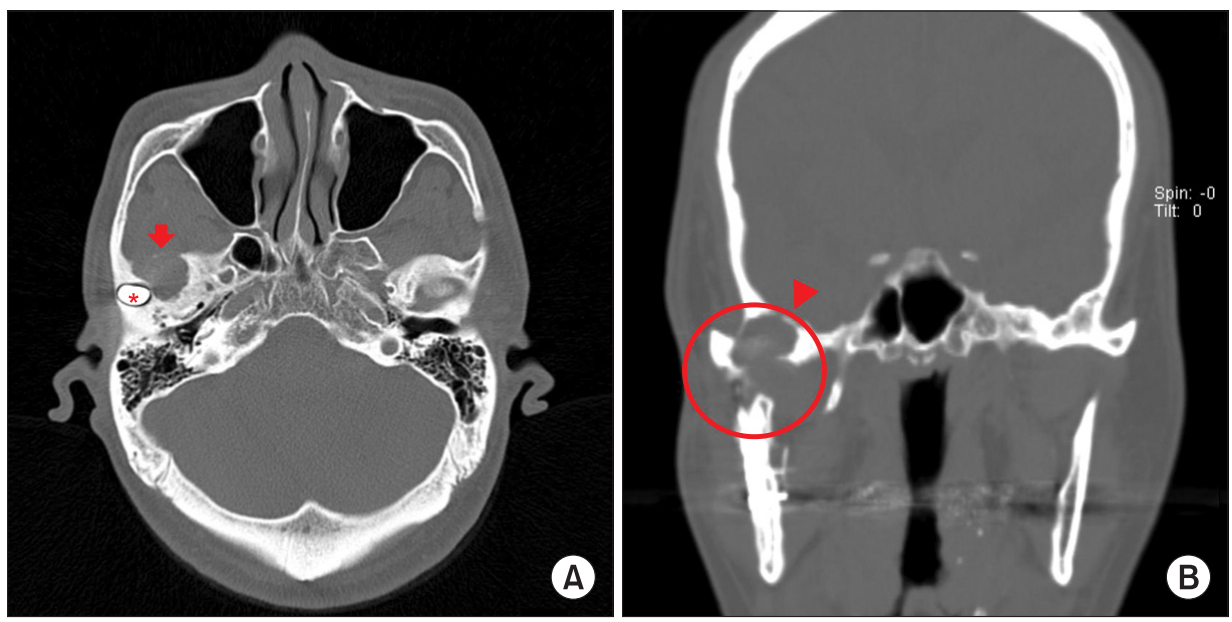

Fig. 9. The axial (A) and coronal (B) views of computed tomography show the round soft tissue opacity on the condylar fossa of temporal bone (arrow) and the skull base erosion with bony perforation (arrow head). Asterisk: metal condylar head.

Il-Kyu Kim et al: Pigmented villonodular synovitis of the temporomandibular joint - computed tomography and magnetic resonance findings: a case report. J Korean Assoc Oral Maxillofac Surg 2014
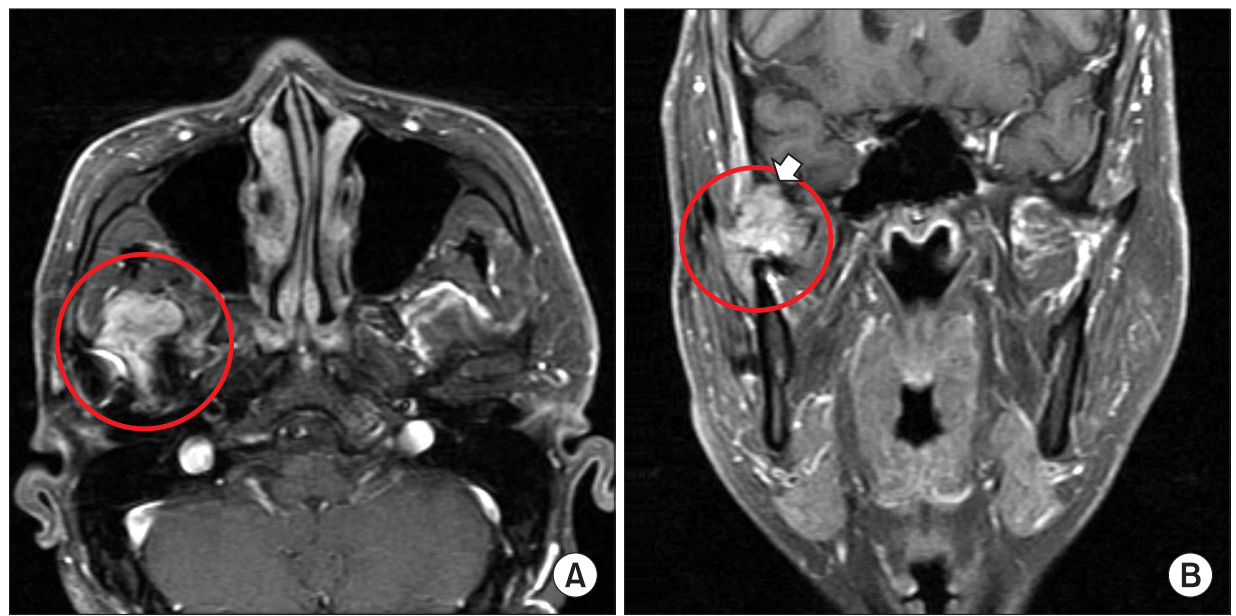

Fig. 10. The axial $(A)$ and coronal $(B)$ fat suppression $\mathrm{T} 1$-weighted images of magnetic resonance imaging show the lobulated high signal intensity mixed up inner intermediate signal intensity with the moderate enhancement. The skull base perforation (arrow) is also seen. Il-Kyu Kim et al: Pigmented villonodular synovitis of the temporomandibular joint - computed tomography and magnetic resonance findings: a case report. J Korean Assoc Oral Maxillofac Surg 2014 
volved the mandibular condyle, which was eroded and perforated on its posterior surface.(Fig. 7) Grossly, the specimen consisted of a few fragments of brownish gray soft tissue, cartilage, and bone. Microscopically, the tumor was composed of many mononuclear histiocytic cells and irregularly interspersed multinucleated giant cells. Hemosiderin pigments were also found. Some foamy histiocytic cells were individually interspersed or formed clusters.(Fig. 8) Osseous, cartilaginous, and soft tissue involvement was also seen. The lesion was diagnosed as a PVNS with involvement of the bone, cartilage, and adjacent soft tissue.

The 4-year follow-up CT and MRI examination revealed a recurrence of the PVNS with a $2.3 \times 2.1 \times 2.6 \mathrm{~cm}$ mass. Ero-

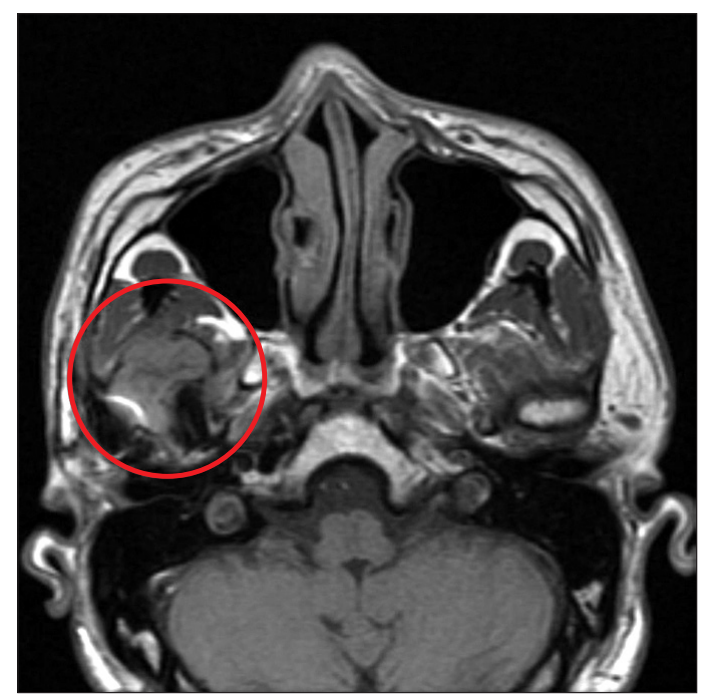

Fig. 11. The axial T1-weighted image of magnetic resonance imaging shows the isosignal intensity mixed up inner low signal intensity with peripheral low signal intensity rim.

Il-Kyu Kim et al: Pigmented villonodular synovitis of the temporomandibular jointcomputed tomography and magnetic resonance findings: a case report. J Korean Assoc Oral Maxillofac Surg 2014 sion of the skull base of the temporal bone was noticed to accompany the perforation.(Figs. 9, 10) The findings on MRI were similar with the preoperative signal intensities of the T1W1, T2W1, and fat suppression T1W1 images.(Figs. 1012) Surgery was performed again on July 2008 and the recurrence of PVNS was confirmed. During the follow-up with $\mathrm{CT}$ and MRI examination, fracture of the reconstruction plate was noticed, with the patient complaining of some discomfort and a limitation in mouth opening. In December 2012, total joint reconstruction with Biomet stock prosthesis (Biomet, Jacksonvill, FL, USA) was performed, and biopsy of the excised soft tissue from the old lesion revealed fibrosis with hyalinization and mild inflammation. There were no clinical or imaging signs of recurrence after 18 months of follow-up following the final operation.(Fig. 13)

\section{Discussion}

PVNS is an idiopathic proliferative disorder involving the synovium of the joint, tendon sheaths, and bursae. More

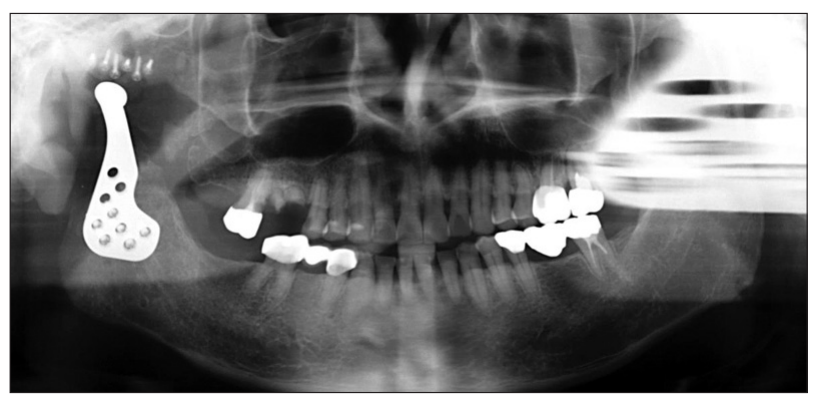

Fig. 13. The panoramic view after 18 months of final operation later.

Il-Kyu Kim et al: Pigmented villonodular synovitis of the temporomandibular jointcomputed tomography and magnetic resonance findings: a case report. J Korean Assoc Oral Maxillofac Surg 2014
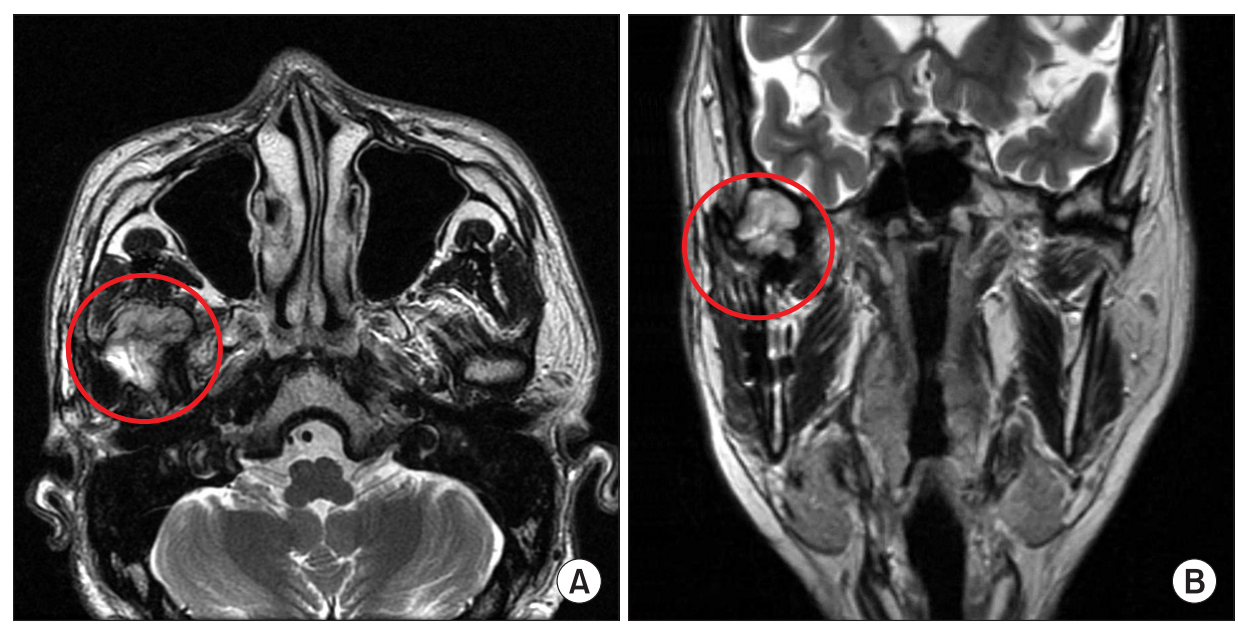

Fig. 12. The axial (A) and coronal (B) T2-weighted images of magnetic resonance imaging show the heterogenous high signal intensity mixed up inner low signal intensity with peripheral low signal intensity rim.

Il-Kyu Kim et al: Pigmented villonodular synovitis of the temporomandibular joint - computed tomography and magnetic resonance findings: a case report. J Korean Assoc Oral Maxillofac Surg 2014 
than $80 \%$ of PVNS cases involve the knees and the upper extremities are seldom involved, suggesting a predilection for weight-bearing joints. Eighty-eight percentage of localized PVNS and 59\% of diffuse PVNS have a positive traumatic history. Bloody fluid was obtained in most of the cases undergoing synovial fluid aspiration, and yellow fluid was obtained in a few cases ${ }^{7,8}$.

Reports of PVNS in the TMJ are rare, but PVNS can occur at any age, with a previous incidence peak in the fourth and fifth decades of life. No gender difference is seen ${ }^{9}$. When the disease does occur in the TMJ, it usually arises as a preauricular palpable mass or swelling, and is frequently mistaken for a parotid gland lesion. Other symptoms may include a restricted mandibular range of motion, stiffness, numbness, and pain. In most cases, the pain was of minimal intensity. A review of the literature and careful analysis of the clinicopathologic features of PVNS of the TMJ reveal that it typically occurs in the diffuse form, and the vast majority of reported cases were of the extra-articular variant of this disease, which is associated with more aggressive local infiltrative behavior, such as involving the parotid gland or skull base, and a higher rate of local recurrence than the localized type $e^{6,10,11}$. The patient in this study was thought to have the diffuse form, and an aggressive local recurrence with skull base perforation by bony erosion sparing the dura was found 4 years after the operation.

The etiology of PVNS still remains controversial. While Jaffe et al. ${ }^{1}$ originally thought it was an inflammatory response to an unknown stimulus, others have attributed the process to repetitive intra-articular hemorrhage following trauma, disturbances in lipid metabolism, or a benign neoplastic proliferation ${ }^{7,9}$. The patient in this study did not recall a history of facial trauma.

The macroscopic features of PVNS include a thick synovium comprised of matted masses of villi and synovial folds, or pedunculated nodules. The lesion has a characteristic rusty, red-brown color resulting from extensive hemosiderin deposition. The microscopic features of PVNS indicate a nonspecific inflammatory process, resulting in a papillary, villous, and nodular appearance of the stratified synovium with multinucleated giant cells, foam cells, and hemosiderin deposition $^{3,9}$.

The classic plain radiographic findings of PVNS consist of cystic erosions, increased density of the synovium secondary to hemosiderin deposition, and periarticular soft-tissue swelling with no calcification ${ }^{5,9}$. CT clearly reveals areas of lytic bone erosion and sclerosis. CT also defines the extent of the tumor well because the tumor has all or most of its focal areas of hyperdensity within the soft-tissue mass that further enhances after administration of contrast material. CT enhances the thickened synovium and areas of high attenuation caused by iron deposits ${ }^{11-13}$. In this patient, CT also showed a lobulated osteolytic lesion accompanying the bony erosion, but did not find bony sclerosis and areas of focal hyperdensity within the soft tissue.(Figs. 2, 9)

The appearance of PVNS on MR image can be variable, depending on the relative proportion of lipids, hemosiderin, fibrous stroma, pannus, fluids, and cellular elements ${ }^{2,13}$. For example, depending on the degree of hemosiderin deposition, tumors may demonstrate low signal intensity on T1-, T2-, and proton density-weighted sequences; the low signal intensity is the result of the presence of hemosiderin deposition. Hemosiderin typically decreased signal intensity on longrepetition time and long-echo time spin-echo (SE) sequences largely due to the ferromagnetic properties of hemosiderin. On T2-weighted SE and gradient-echo (GRE) images, the abnormal tissue may appear larger because of so-called blooming. On the other hand, the accumulation of lipids in foamy macrophages may produce areas of high signal intensity on T1-weighted SE images similar to subcutaneous fat. Additionally, areas of high signal intensity on T2-weighted images correspond to a loculated cyst of joint fluid. Another well-recognized characteristics of PVNS on MR images are hyperplastic synovium and bone erosion in the joint with insufficient intra-articular space for outward proliferation of the synovium. In this patient, the $3.0 \times 2.5 \mathrm{~cm}$ irregularly shaped mass had heterogeneous T2 signal intensity and isosignal intensity on T1-weighted MRI. Peripheral low signal intensity areas are noted on both T1- and T2-weighted MR images. (Figs. 3-5, 10-12) In the present case, low signal intensity on T1-weighted sequences was also observed (Figs. 4, 11), but the causes of heterogeneous high signal intensity on T2weighted and fat suppression T1 MR images on the mass were thought to be due to the profound fluid from the cystic lesion from the condyle to the synovium.(Figs. 3, 5, 10, 12)

Treatment of PVNS is exclusively surgical and consists of complete excision of the involved bone and total synovectomy because synovial lesions have a high rate of local recurrence. The recurrence of TMJ PVNS occurs primarily in the diffuse type in $8 \%$ to $46 \%$ of cases and usually results from incomplete resection, but can also be spontaneous ${ }^{14}$. Radiation therapy may produce involution of the lesion but should be reserved for only those recurrent cases with involvement of vital structures ${ }^{15}$. 


\section{Conflict of Interest}

No potential conflict of interest relevant to this article was reported.

\section{References}

1. Jaffe HL, Lichtenstein L, Sutro CJ. Pigmented villonodular synovitis, bursitis and tenosynovitis. Arch Pathol 1941;31:731-65.

2. Kim KW, Han MH, Park SW, Kim SH, Lee HJ, Jae HJ, et al. Pigmented villonodular synovitis of the temporomandibular joint: MR findings in four cases. Eur J Radiol 2004;49:229-34.

3. Aoyama S, Iwaki H, Amagasa T, Kino K, Okada N, Kishimoto S. Pigmented villonodular synovitis of the temporomandibular joint: differential diagnosis and case report. Br J Oral Maxillofac Surg 2004;42:51-4.

4. Kişnişci RS, Tüz HH, Günhan O, Onder E. Villonodular synovitis of the temporomandibular joint: case report. J Oral Maxillofac Surg 2001;59:1482-4.

5. Lapayowker MS, Miller WT, Levy WM, Harwick RD. Pigmented villonodular synovitis of the temperomandibular joint. Radiology 1973;108:313-6.

6. Liu YK, Chan JY, Chang CJ, Huang JS. Pigmented villonodular synovitis of the temporomandibular joint presenting as a middle cranial fossa tumor. J Oral Maxillofac Surg 2012;70:367-72.

7. Bemporad JA, Chaloupka JC, Putman CM, Roth TC, Tarro J, Mi- tra S, et al. Pigmented villonodular synovitis of the temporomandibular joint: diagnostic imaging and endovascular therapeutic embolization of a rare head and neck tumor. AJNR Am J Neuroradiol 1999;20:159-62.

8. Cai J, Cai Z, Gao Y. Pigmented villonodular synovitis of the temporomandibular joint: a case report and the literature review. Int $\mathrm{J}$ Oral Maxillofac Surg 2011;40:1314-22.

9. Cascone P, Rinna C, Ungari C, Poladas G, Filiaci F. Pigmented villonodular synovitis of the temporomandibular joint. J Craniofac Surg 2005;16:712-6.

10. Safaee M, Oh T, Sun MZ, Parsa AT, McDermott MW, El-Sayed $\mathrm{IH}$, et al. Pigmented villonodular synovitis of the temporomandibular joint with intracranial extension: a case series and systematic review. Head Neck 2014. doi: 10.1002/hed.23717.

11. Church CA, Rowe M, Llaurado R, Liwnicz BH, Martin PA. Pigmented villonodular synovitis of the temporomandibular joint: a report of two cases. Ear Nose Throat J 2003;82:692-5.

12. Le WJ, Li MH, Yu Q, Shi HM. Pigmented villonodular synovitis of the temporomandibular joint: CT imaging findings. Clin Imaging 2014;38:6-10.

13. Stryjakowska KK, Martel M, Sasaki CT. Pigmented villonodular synovitis of the temporomandibular joint: differential diagnosis of the parotid mass. Auris Nasus Larynx 2005;32:309-14.

14. Wong JJ, Phal PM, Wiesenfeld D. Pigmented villonodular synovitis of the temporomandibular joint: a radiologic diagnosis and case report. J Oral Maxillofac Surg 2012;70:126-34.

15. O'Sullivan TJ, Alport EC, Whiston HG. Pigmented villonodular synovitis of the temporomandibular joint. J Otolaryngol 1984;13:123- 